\title{
(2+1)-flavor QCD Thermodynamics from the Gradient Flow*
}

\author{
Etsuko Itou ${ }^{\dagger}$ \\ Theory Center, High Energy Accelerator Research Organization (KEK) \\ E-mail: eitoudpost.kek.ip
}

\section{Hiroshi Suzuki}

Department of Physics, Kyushu University, 744 Motooka, Nishi-ku, Fukuoka, 812-0395, Japan

E-mail: hsuzukiephys.kyushu-u.ac.jp

\section{Yusuke Taniguchi}

Graduate School of Pure and Applied Sciences, University of Tsukuba, Tsukuba, Ibaraki

305-8571, Japan

E-mail: taniqchiehet.ph.tsukuba.ac.jp

\section{Takashi Umeda}

Graduate School of Education, Hiroshima University, Hiroshima 739-8524, Japan

E-mail: tumedadhiroshima-u.ac.jp

Recently, we proposed a novel method to define and calculate the energy-momentum tensor (EMT) in lattice gauge theory on the basis of the Yang-Mills gradient flow [四]. In this proceedings, we summarize the basic idea and technical steps to obtain the bulk thermodynamic quantities in lattice gauge theory using this method for the quenched and $(2+1)$-flavor QCD. The revised results of integration measure (trace anomaly) and entropy density of the quenched QCD with corrected coefficients are shown. Furthermore, we also show the flow time dependence of the parts of EMT including the dynamical fermions. This work is based on a joint-collaboration between FlowQCD and WHOT QCD.

The 33rd International Symposium on Lattice Field Theory

14 -18 July 2015

Kobe International Conference Center, Kobe, Japan*

\footnotetext{
${ }^{*}$ Numerical simulation for this study was carried out on Hitachi SR16000 and IBM System Blue Gene Solution at KEK under its Large-Scale Simulation Program (No. 14/15-11), Hitachi SR16000 at YITP Kyoto University and NEC SX-8 and SX-9 at RCNP Osaka University. E. I. is supported in part by Strategic Programs for Innovative Research (SPIRE) Field 5. H. S. and T. U. are in supported by the Japan Society for the Promotion of Science (JSPS) Grants Number 23540330 and 26400251 , respectively.

† Speaker.
} 


\section{Introduction}

Measurement of the energy-momentum tensor (EMT) that is a generator of the general coordinate transformation is difficult using the lattice numerical simulation, since the lattice regularization manifestly breaks the corresponding invariance. Recently, we proposed a novel method to obtain the EMT using the lattice numerical simulation [W] based on the small flow-time expansion of the Yang-Mills gradient flow [ [ , []]. As a first observation, the bulk thermal quantities, namely integration measure (trace anomaly) and thermal entropy density, are calculated by the direct measurement of EMT. In this proceedings, we review the quenched QCD results, and also show the detailed strategy and a preliminary result for $(2+1)$-flavor QCD simulation.

\section{Quenched QCD}

\section{1 strategy}

A key property of the Yang-Mills gradient flow [ฉ] is UV finiteness of local operators [ [ 7 ]. For example, the following gauge-invariant local products of dimension 4 are UV finite for the positive flow-time $(t>0): U_{\mu \nu}(t, x) \equiv G_{\mu \rho}(t, x) G_{\nu \rho}(t, x)-\frac{1}{4} \delta_{\mu \nu} G_{\rho \sigma}(t, x) G_{\rho \sigma}(t, x)$ and $E(t, x) \equiv$ $\frac{1}{4} G_{\mu \nu}(t, x) G_{\mu v}(t, x)$. Here $G_{\mu \nu}$ presents the field strength constructed by the flowed gauge field.

The expansion coefficients are governed by the renormalization group equation and their small $t$ behavior can be calculated by perturbation theory thanks to the asymptotic freedom. For the operators mentioned above, we have [3], 可]

$$
\begin{aligned}
U_{\mu v}(t, x) & =\alpha_{U}(t)\left[T_{\mu v}^{R}(x)-\frac{1}{4} \delta_{\mu v} T_{\rho \rho}^{R}(x)\right]+O(t), \\
E(t, x) & =\langle E(t, x)\rangle_{0}+\alpha_{E}(t) T_{\rho \rho}^{R}(x)+O(t),
\end{aligned}
$$

where $\langle\cdot\rangle_{0}$ is vacuum expectation value (v.e.v.) and $T_{\mu \nu}^{R}(x)$ is the correctly-normalized conserved EMT with its vacuum expectation value subtracted. Abbreviated are the contributions from the operators of dimension 6 or higher, which are suppressed for small $t$.

In $t \rightarrow 0$ limit, the correctly-normalized EMT is given by

$$
T_{\mu v}^{R}(x)=\lim _{t \rightarrow 0}\left\{\frac{1}{\alpha_{U}(t)} U_{\mu v}(t, x)+\frac{\delta_{\mu v}}{4 \alpha_{E}(t)}\left[E(t, x)-\langle E(t, x)\rangle_{0}\right]\right\}
$$

where the perturbative coefficients are found to be [[] $]$

$$
\begin{aligned}
& \alpha_{U}(t)=\bar{g}(1 / \sqrt{8 t})^{2}\left[1+2 b_{0} \bar{s}_{1} \bar{g}(1 / \sqrt{8 t})^{2}+O\left(\bar{g}^{4}\right)\right], \\
& \alpha_{E}(t)=\frac{1}{2 b_{0}}\left[1+2 b_{0} \bar{s}_{2} \bar{g}(1 / \sqrt{8 t})^{2}+O\left(\bar{g}^{4}\right)\right] .
\end{aligned}
$$

Here $\bar{g}(q)$, which is the running gauge coupling constant, and the coefficients $\bar{s}_{1}$ and $\bar{s}_{2}$ depend on the renormalization scheme. In the $\overline{\mathrm{MS}}$ scheme with the scale $q=1 / \sqrt{8 t}, \bar{s}_{1}=\frac{7}{22}+\frac{1}{2} \gamma_{E}-\ln 2 \simeq$ $-0.08635752993, \bar{s}_{2}=\frac{21}{44}-\frac{b_{1}}{2 b_{0}^{2}}=\frac{27}{484} \simeq 0.05578512397,{ }^{1}$ with $b_{0}=\frac{1}{(4 \pi)^{2}} \frac{11}{3} N_{c}, b_{1}=\frac{1}{(4 \pi)^{4}} \frac{34}{3} N_{c}^{2}$, and $N_{c}=3$.

\footnotetext{
${ }^{1}$ Note that in the published version of our paper [四] the values of these coefficients were wrong. Corrected results are shown in Ver.3 on arXiv.
} 
Our procedure to calculate the EMT on the lattice has the following four steps:

Step 1: Generate gauge configurations at $t=0$ on a space-time lattice with the lattice spacing $a$ and the lattice size $N_{s}^{3} \times N_{\tau}$.

Step 2: Solve the gradient flow for each configuration to obtain the flowed link variables in the fiducial window, $a \ll \sqrt{8 t} \ll R$. Here, $R$ is an infrared cutoff scale such as $\Lambda_{\mathrm{QCD}}^{-1}$ or $T^{-1}=N_{\tau} a$. The first (second) inequality is necessary to suppress finite $a$ corrections (non-perturbative corrections and finite volume corrections).

Step 3: Construct $U_{\mu v}(t, x)$ and $E(t, x)$ in Eqs. (1.2) and (2.2) in terms of the flowed link variables and average over the gauge configurations at each $t$.

Step 4: Carry out an extrapolation toward $(a, t)=(0,0)$, first $a \rightarrow 0$ and then $t \rightarrow 0$ under the condition in Step 2.

\section{2 results}

We consider the pure $S U(3)$ gauge theory defined on a four-dimensional Euclidean lattice,

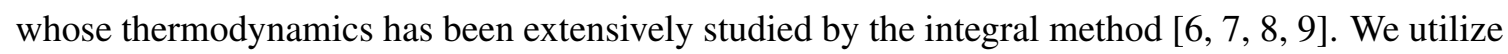
the Wilson plaquette gauge action under the periodic boundary condition on $N_{s}^{3} \times N_{\tau}=32^{3} \times$ $(6,8,10)$ lattices with several different $\beta=6 / g_{0}^{2}$ ( $g_{0}$ being the bare coupling constant) shown in Table $\mathbb{W}$. These lattice parameters are determined by the relation between the Sommer scale and $\beta$ given by ALPHA collaboration [ए]] and the critical temperature $\left(T_{c}\right)$ given in Ref. [四]. Gauge configurations are generated by the pseudo-heatbath algorithm with the over-relaxation, mixed in the ratio of $1: 5$. We call one pseudo-heatbath update sweep plus five over-relaxation sweeps as a "Sweep". To eliminate the autocorrelation, we take 200-500 Sweeps between measurements. The number of gauge configurations for the measurements at finite $T$ is $100-300$. Statistical errors are estimated by the jackknife method.

\begin{tabular}{|c||c|c|c||c|}
\hline$N_{\tau}$ & 6 & 8 & 10 & $T / T_{c}$ \\
\hline & 6.20 & 6.40 & 6.56 & 1.65 \\
$\beta$ & 6.02 & 6.20 & 6.36 & 1.24 \\
& 5.89 & 6.06 & 6.20 & 0.99 \\
\hline
\end{tabular}

Table 1: Values of $\beta$ and $N_{\tau}$ for each temperature.

The left panel of Fig. $\square$ is our results for the dimensionless interaction measure $\left(\Delta / T^{4}=(\varepsilon-\right.$ $\left.3 P) / T^{4}\right)$ and the dimensionless entropy density $\left(s / T^{3}=(\varepsilon+P) / T^{4}\right)$ at $T=1.65 T_{c}$ as a function of the dimensionless flow parameter $\sqrt{8 t} T$. The bold bars denote the statistical errors, while the thin (light color) bars show the statistical and systematic errors including the uncertainty of $\Lambda_{\overline{\mathrm{MS}}}$. In the small $t$ region, the statistical error is dominant for both $\Delta / T^{4}$ and $s / T^{3}$, while in the large $t$ region the systematic error from $\Lambda_{\overline{\mathrm{MS}}}$ becomes significant for $s / T^{3}$. For instance, the statistical (systematic) errors of the data for $N_{\tau}=8$ are $2.5 \%(0.11 \%)$ for $\Delta / T^{4}$ and $0.83 \%$ (4.4\%) for $s / T^{3}$ at $\sqrt{8 t} T=0.40$.

The fiducial window discussed in Step 2 is indicated by the dashed lines in left panel of Fig. . The lower limit, beyond which the lattice discretization error grows, is set to be $\sqrt{8 t_{\min }}=2 a$, where 

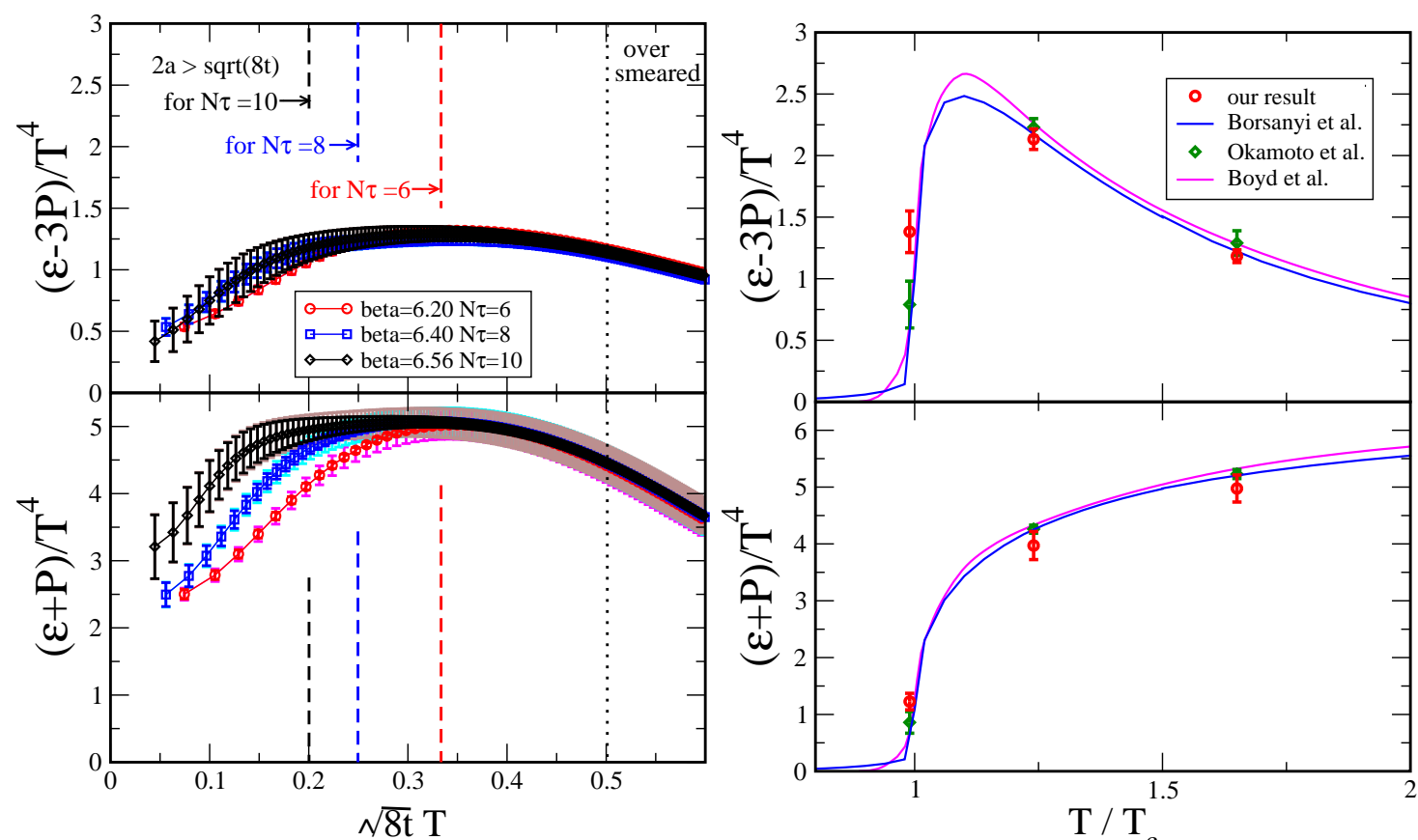

Figure 1: (left)Flow time dependence of the dimensionless interaction measure (top panel) and the dimensionless entropy density (bottom panel) for different lattice spacings at fixed $T / T_{c}=1.65$. The circles (red) the squares (blue), and the diamonds (black) correspond to $N_{\tau}=6,8$, and 10, respectively. The bold error bars denote the statistical errors, while the thin error bars (brown, cyan, and magenta) include both statistical and systematic errors. (right)Continuum limit of the interaction measure and entropy density obtained by the gradient flow for $T / T_{c}=1.65,1.24$, and 0.99 with 300 gauge configurations. The magenta, green, and blue data are the results of the integral method according to Ref. [G, $\square$, Q $]$ ], respectively

we consider the size $2 a$ of our clover leaf operator. The upper limit, beyond which the smearing by the gradient flow exceeds the temporal lattice size, is set to be $\sqrt{8 t_{\max }}=1 /(2 T)=N_{\tau} a / 2$.

Finally, we plot, in the right panel of Fig. $\mathbb{1}, \Delta / T^{4}$ and $s / T^{3}$ after taking the continuum limit using the linear fit of the $N_{\tau}=6,8$, and 10 data for $T / T_{c}=1.65,1.24$, and 0.99 . For comparison, the results of Ref. [ $[\square, \square, 0]$ obtained by the integral method are shown by the magenta, green, and

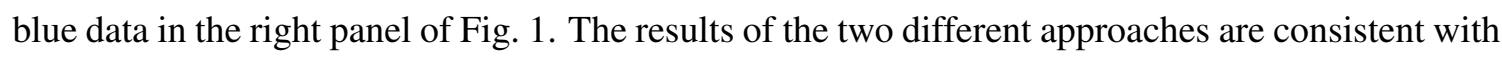
each other within the statistical error.

\section{3. (2+1)-flavor QCD}

\section{1 strategy}

Now, let us extend the previous method to the full QCD system. One property, which is no necessity of wave function renormalization factor of composite operators, is lost for the dynamical fermion system. We have to take care of the renormalization for fermion fields. Except for this and several trivial technical steps, the previous small flow-time expansion can straightforwardly apply to the full QCD system. 
Firstly, the fermion flow equation in terms of the Wilson flow-time must be solved in the case of full QCD. The equation is given by

$$
\begin{aligned}
& \partial_{t} \chi^{f}(t, x)=\Delta \chi^{f}(t, x), \quad \chi^{f}(t=0, x)=\psi^{f}(x), \\
& \partial_{t} \bar{\chi}^{f}(t, x)=\bar{\chi}^{f}(t, x) \overleftarrow{\Delta}, \quad \bar{\chi}^{f}(t=0, x)=\bar{\psi}^{f}(t, x),
\end{aligned}
$$

where $f$ denotes a label of the quark flavor and $\Delta \chi^{f}(t, a)=D_{\mu} D_{\mu} \chi^{f}(t, x)$. Note that the covariant derivative refers to the flowed gauge field at the flow time $t$.

To obtain the thermal bulk quantity, which is independent of the imaginary time, introducing the random source fields and solving the adjoint flow equation proposed in Ref. [U]] are useful.

The EMT for the full QCD in the small flow-time limit is given by five dimension-4 operators in Ref. [12] as follows:

$$
\begin{aligned}
T_{\mu v}^{R}(x)= & \lim _{t \rightarrow 0}\left\{c_{1}(t)\left[O_{1 \mu v}(t, x)-\frac{1}{4} O_{2 \mu v}(t, x)\right]\right. \\
& +c_{2}(t)\left[O_{2 \mu v}(t, x)-\left\langle O_{2 \mu v}(t, x)\right\rangle\right] \\
& +c_{3}(t) \sum_{f=u, d, s}\left[O_{3 \mu v}^{f}(t, x)-2 O_{4 \mu v}^{f}(t, x)-\left\langle O_{3 \mu v}^{f}(t, x)-2 O_{4 \mu v}^{f}(t, x)\right\rangle\right] \\
& +c_{4}(t) \sum_{f=u, d, s}\left[O_{4 \mu v}^{f}(t, x)-\left\langle O_{4 \mu v}^{f}(t, x)\right\rangle\right] \\
& \left.+c_{5}^{f}(t) \sum_{f=u, d, s}\left[O_{5 \mu v}^{f}(t, x)-\left\langle O_{5 \mu v}^{f}(t, x)\right\rangle\right]\right\} .
\end{aligned}
$$

The scalar coefficients $c_{1}(t)-c_{5}^{f}(t)$ within 1loop order are given by the running coupling constant and running masses for each flavor and explicitly shown in Eqs.(4.60)-(4.64) in Ref. [12]]. Note that the $c_{5}^{f}(t)$, which is related with the quark mass term, depends on the flavor. The explicit form of each dimension- 4 operator are given by

$$
\begin{aligned}
O_{1 \mu v}(t, x) & \equiv G_{\mu \rho}^{a}(t, x) G_{\rho v}^{a}(t, x) \\
O_{2 \mu v}(t, x) & \equiv \delta_{\mu v} G_{\rho \sigma}^{a}(t, x) G_{\rho \sigma}^{a}(t, x) \\
O_{3 \mu v}^{f}(t, x) & \equiv \varphi^{f}(t) \bar{\chi}^{f}(t, x)\left(\gamma_{v} \stackrel{\leftrightarrow}{D}_{v}+\gamma_{v} \stackrel{\leftrightarrow}{D}_{\mu}\right) \chi^{f}(t, x) \\
O_{4 \mu v}^{f}(t, x) & \equiv \varphi^{f}(t) \delta_{\mu v} \bar{\chi}^{f}(t, x) \varkappa_{\rho} \stackrel{\leftrightarrow}{D}_{\rho} \chi^{f}(t, x) \\
O_{5 \mu v}^{f}(t, x) & \equiv \varphi^{f}(t) \delta_{\mu v} \bar{\chi}^{f}(t, x) \chi^{f}(t, x)
\end{aligned}
$$

where $\stackrel{\leftrightarrow}{D}_{\mu} \equiv D_{\mu}-\overleftarrow{D}_{\mu}$. The coefficient $\varphi(t)$ is introduced to cancel the renormalization factor for the fermion and defined by

$$
\varphi^{f}(t) \equiv \frac{-6}{(4 \pi)^{2} t^{2}\left\langle\bar{\chi}^{f}(t, x) \stackrel{\leftrightarrow}{D}_{\mu} \gamma_{\mu} \chi^{f}(t, x)\right\rangle}
$$

This is numerically calculated by the lattice configurations at zero temperature simulation.

In full QCD case, three dimension-4 operators, $\mathrm{O}_{3}-\mathrm{O}_{5}$, are added. To obtain the thermal quantities from the EMT, we additionally compute these expectation values. These values can be 
summarized two types of expectation value as follows;

$$
\begin{aligned}
t_{\mu v}^{f}(t) & \equiv \frac{1}{N_{\Gamma}} \sum_{x}\left\langle\bar{\chi}^{f}(t, x) \gamma_{\mu}\left(D_{v}-\overleftarrow{D}_{v}\right) \chi^{f}(t, x)\right\rangle \\
s^{f}(t) & \equiv \frac{1}{N_{\Gamma}} \sum_{x}\left\langle\bar{\chi}^{f}(t, x) \chi^{f}(t, x)\right\rangle
\end{aligned}
$$

where $N_{\Gamma}$ is the lattice volume in lattice unit.

Now, let us summarize the procedure to calculate the EMT on the lattice for $(2+1)$-flavor QCD:

Step 1: Generate gauge configurations at $t=0$ on a space-time lattice with the lattice spacing $a$ and the lattice size $N_{s}^{3} \times N_{\tau}$ with dynamical fermions

Step 2: Solve both the gradient flow for the link variable and the adjoint fermion flow to obtain the flowed field.

Step 3-1: Construct $U_{\mu v}(t, x)$ and $E(t, x)$ in Eqs. ([2. $(1)$ and (2.2) in terms of the flowed link variables and average over the gauge configurations at each $t$.

Step 3-2: Calculate $t_{\mu v}(t, x), s(t, x)$ and $\varphi(t)$ in Eqs. (B.5), (B.6) and (B. (B) $)$ using the flowed quark fields and average over the gauge configurations at each $t$, respectively.

Step 4: Carry out an extrapolation toward $(a, t)=(0,0)$, first $a \rightarrow 0$ and then $t \rightarrow 0$ within the fiducial window of the flow time.

\subsection{Simulation details and preliminary results}

We performed QCD with $(2+1)$-flavor of quarks. The Iwasaki gauge action and the standard $O(a)$-improved Wilson fermion are used in the simulation. The lattice parameters are determined based on Ref. [[3]]. The hopping parameter is tuned to realize $m_{P S} / m_{V}=0.6337$ for $u, d$ quarks and $m_{P S} / m_{V}=0.7377$ for $s$ quark. The lattice extent is $N_{s}^{3} \times N_{\tau}=32^{3} \times 8$ and the lattice bare gauge coupling and $c_{S W}$ are $\beta=1.973$ and $c_{S W}=1.669$. The temperature is estimated to be $280 \mathrm{MeV}$.

The number of configuration is still 11, where we take 100 Montecarlo trajectories between measurements. The error bar denotes the standard statistical error.

Figure $\square$ shows the preliminary results for $t_{\mu v}(t)$ and $s(t)$ in Eqs. (B.5) and (B.6). The circle (black) and square (red) symbol present $u, d$ and $s$ quarks on each panel, respectively. Here, $s(t)$ is the chiral condensate. It decreases to almost zero as expected, since the present temperature is higher than $T_{c}$.

The statistical error is a few $\%$ order in these operators with only 11 configurations. We consider that the method looks promising to obtain the thermal quantities even in the full QCD simulation, although the careful estimation of the autocorrelation should be done. Further simulations will give a precise determination of them in near future.

\section{Acknowedgement}

We would like to thank M. Asakawa, T. Hatsuda, T. Iritani and M. Kitazawa for useful discussions. We are also grateful to K. Kanaya and H. Matsufuru for their helps of the code development. 


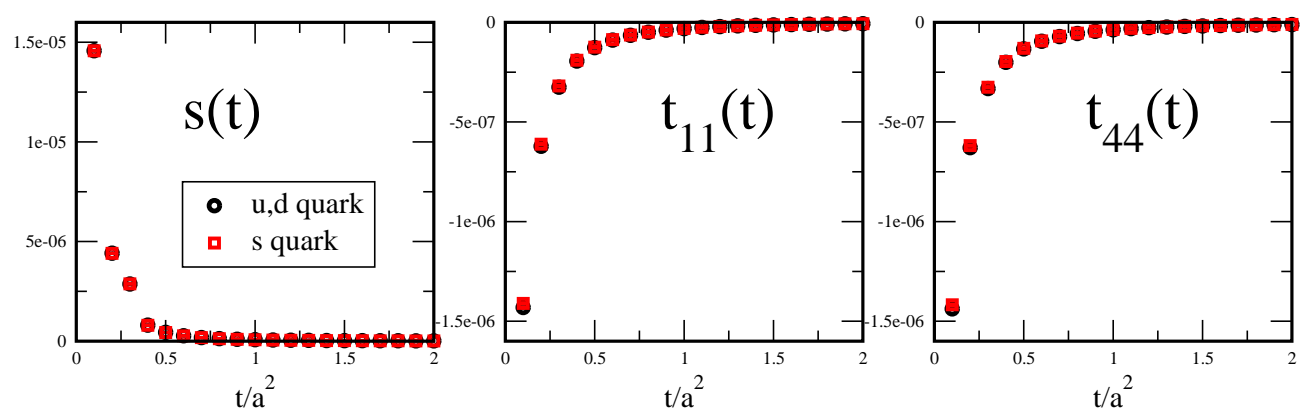

Figure 2: Flow time dependence of the $s(t)$ (left), $t_{11}$ (middle) and $t_{44}$ (right) with $\beta=1.973, c_{S W}=1.669$, $\kappa_{u, d}=0.1361, \kappa_{s}=0.1354$ on $32^{3} \times 8$ lattices. Circle (black) and square (red) symbols denote $u, d$ and $s$ quarks on each panel, respectively.

\section{References}

[1] M. Asakawa et al. [FlowQCD Collaboration], Phys. Rev. D 90, no. 1, 011501 (2014) [Phys. Rev. D 92, no. 5, 059902 (2015)] [arXiv:1312.7492 [hep-lat]].

[2] M. Lüscher, JHEP 1008, 071 (2010) [arXiv:1006.4518 [hep-lat]].

[3] H. Suzuki, PTEP 2013, 083B03 (2013) [PTEP 2015, 079201 (2015)] [arXiv:1304.0533 [hep-lat]].

[4] M. Lüscher and P. Weisz, JHEP 1102, 051 (2011) [arXiv:1101.0963 [hep-th]].

[5] L. Del Debbio, A. Patella and A. Rago, JHEP 1311, 212 (2013) [arXiv:1306.1173 [hep-th]].

[6] G. Boyd, J. Engels, F. Karsch, E. Laermann, C. Legeland, M. Lutgemeier and B. Petersson, Nucl. Phys. B 469, 419 (1996) [hep-lat/9602007].

[7] M. Okamoto et al. [CP-PACS Collaboration], Phys. Rev. D 60, 094510 (1999) [hep-lat/9905005]; Y. Namekawa et al. [CP-PACS Collaboration], Phys. Rev. D 64, 074507 (2001).

[8] T. Umeda, S. Ejiri, S. Aoki, T. Hatsuda, K. Kanaya, Y. Maezawa and H. Ohno, Phys. Rev. D 79, 051501 (2009) [arXiv:0809.2842 [hep-lat]].

[9] S. Borsanyi, G. Endrodi, Z. Fodor, S. D. Katz and K. K. Szabo, JHEP 1207, 056 (2012) [arXiv:1204.6184 [hep-lat]].

[10] M. Guagnelli et al. [ALPHA Collaboration], Nucl. Phys. B 535, 389 (1998) [hep-lat/9806005].

[11] M. Lüscher, JHEP 1304, 123 (2013) [arXiv:1302.5246 [hep-lat]].

[12] H. Makino and H. Suzuki, PTEP 2014, $063 B 02$ (2014) [PTEP 2015, 079202 (2015)] [arXiv:1403.4772 [hep-lat]].

[13] T. Umeda et al. [WHOT-QCD Collaboration], Phys. Rev. D 85, 094508 (2012) [arXiv:1202.4719 [hep-lat]]. 